\title{
Proteolysis in the lung
}

\author{
S.D. Shapiro
}

Pulmonary emphysema is a major component of the morbidity and mortality of chronic obstructive pulmonary disease (COPD), a condition that has become the fourth leading cause of death in the USA, and is becoming epidemic worldwide. Emphysema is defined as enlargement of peripheral airspaces of the lung including respiratory bronchioles, alveolar ducts and alveoli, accompanied by destruction of the walls of these structures. The pathogenesis of emphysema can be dissected into three interrelated events: 1) chronic exposure to cigarette smoke may lead to inflammatory cell recruitment within the terminal airspaces of the lung; 2) these inflammatory cells release elastolytic proteinases that damage the extracellular matrix of the lung; and 3) ineffective repair of elastin and perhaps other extracellular matrix components result in pulmonary emphysema.

\section{Proteases in chronic obstructive pulmonary disease}

Inherited deficiency of $\alpha_{1}$-antitrypsin $\left(\alpha_{1}\right.$-AT), the primary inhibitor of neutrophil elastase (NE), predisposes individuals to early onset emphysema, and intrapulmonary instillation of elastolytic enzymes in experimental animals causes emphysema. Together, these findings led to the elastase/antielastase hypothesis for the pathogenesis of emphysema, which was proposed $\sim 40$ yrs ago and remains the prevailing concept today. While the capacity of NE to initiate emphysema in patients deficient in $\alpha_{1}$-AT is clear, the proteinases involved in the pathogenesis of the common form of emphysema associated with cigarette smoking is more complicated. In addition to NE, neutrophil primary granules contain other elastolytic serine proteinases including, cathepsin $G$ and proteinase 3 , and secondary granules possess matrix metalloproteinases; MMP-8, a collagenase and MMP-9, a $92 \mathrm{kDa}$ gelatinase. While, macrophages are prominent inflammatory cells in smokers' lungs, the capacity of macrophages to degrade elastin was controversial until cysteine proteinases including cathepsins $\mathrm{S}$ and $\mathrm{L}$ were identified ( $\mathrm{K}$ the most potent elastase has not been identified in lung macrophages). The author's group identified several MMPs produced by alveolar and interstitial macrophages, including elastolytic MMP-9 and MMP-12.

Expression of most of these proteinases has been associated with human COPD. To determine directly the contribution of MMP-12 to pulmonary emphysema, a murine model of cigarette smoke-induced emphysema was developed, and MMP-12-/- mice and wildtype (MMP-12+/+) littermates were subjected to chronic cigarette smoke exposure. Application of wildtype (MMP-12+/+) mice to long-term cigarette

Correspondence: S.D. Shapiro, Harvard Medical School, Division of Pulmonary and Critical Care, Brigham and Woman's Hospital, 75 Francis Street, 2115 Boston, MA, USA. Fax: 1 6177327421. E-mail: sshapiro@rics.bwh.harvard.edu smoke exposure led to inflammatory cell recruitment followed by alveolar space enlargement similar to the pathological defect in humans. However, mice deficient in macrophage elastase (MMP-12-/-) were protected from development of emphysema despite long-term smoke exposure. MMP-12-/mice also failed to recruit monocytes into their lungs in response to cigarette smoke [1]. Since MMP-12 and most other MMPs are only expressed upon differentiation of monocytes to macrophages, it appeared unlikely that monocytes require MMP-12 for transvascular migration. The working hypothesis is that MMP-12-deficient macrophages have impaired chemotaxis. This could be in part secondary to MMP-12-mediated chemokine activation or generation of elastin fragments, known for many years to be chemotactic for monocytes, and appears to play a role in this condition.

Recently, several other genetically engineered mice have confirmed the capacity of MMPs/MMP-12 to cause airspace enlargement. Inducible, lung-specific transgenic mice expressing either the T-helper cell (Th) type-2 cytokine interleukin$13[2,3]$, or the Th1 cytokine interferon (IFN)- $\gamma[4]$, induce expression of MMP-12, MMP-9, and cysteine proteinases with subsequent airspace enlargement. IFN- $\gamma$-mediated MMP induc-

tion is likely indirect given the finding that IFN- $\gamma$ inhibits macrophage MMP production. WERT et al. [5] found that surfactant protein-D-/- mice demonstrate macrophage activation MMP production and consequent emphysema. Finally, $\alpha_{v} \beta 6-/-$ mice develop macrophage accumulation in the lung, MMP-12 activation and airspace enlargement with age. Emphysema is abrogated upon crossing $\alpha_{\mathrm{v}} \beta 6-/-$ with MMP12-/- mice. This study suggests that transforming growth factor (TGF)- $\beta$ serves to inhibit MMP-12 production under normal circumstances and in the absence of integrin, there is diminished TGF- $\beta$ and enhanced MMP-12 leading to emphysema [6]. Serine proteinases, particularly NE, are also involved. NE-/- mice developed only $40 \%$ as much airspace enlargement as wildtype mice (unpublished data). Several interactions were also found between NE and MMPs. MMPs degrade $\alpha_{1}$-AT and NE degrades tissue inhibitors of metalloproteinases, potentiating the proteinase activity of each other. NE mediates monocyte migration, and MMP-12 may influence acute neutrophil accumulation via tumour necrosis factor- $\alpha$ shedding [7].

Clearly, there are several MMPs expressed in association with emphysema. The major lesson to be gained from the murine model of cigarette smoke-induced emphysema is not that MMP-12 is the sole proteinase responsible for human disease, but that macrophages have the capacity to cause emphysema upon recruitment and activation by cigarette smoke. In mice, the predominant macrophage proteinase is MMP-12; human macrophages probably have a broader spectrum of MMPs (including MMP-12). Moreover, serine proteinases and MMPs interact to augment each others destructive activity. Also, human COPD may be exacerbated by recurrent airway infections. These infections are associated 
with neutrophil recruitment, whose proteinases may also contribute to destructive changes.

\section{Proteinases in cell movement}

A basic function of MMPs has been thought to be to impart the ability of cells to move and to traverse tissue barriers. Tumour cells in particular use these enzymes to clear paths, promoting local tissue invasion and distant metastasis. Extrapolating from these observations of tumour cells, it was speculated that inflammatory cells use their cadre of MMPs to move through tissue barriers in a similar fashion. Indeed, this has been seen to occur.

Yet there has been some confusion, because it was obvious that these enzymes do more than simply clear road-blocks from the path of migrating neutrophils. Moreover, cells have other ways to move. For example, fibroblasts normally take straight paths, degrading the extracellular matrix as they go. However, in the presence of MMP inhibitors, such cells change both their pattern of movement and their shape as they pick their way through microscopic gaps in the matrix. Neutrophils, meanwhile, are known to be the most "flexible" inflammatory cells, able to weave through tissue barriers in the absence of protein-digesting enzymes. Recently, LI et al. [8] have shown that MMP-7 promotes neutrophil migration into the lung following bleomycin "shedding" syndecan-1-KC complexes to form a chemical gradient [8].

To complicate the picture still further, MMPs may also limit inflammation. MCQUIBBAN et al. [9] showed that some of these enzymes process and inactivate the chemokines to which other inflammatory cells (monocytes) respond, thereby restricting monocyte migration into sites of injury. Altogether, this suggests a feedback mechanism whereby MMPs initially promote cell influx to damaged areas, subsequently the excessive quantities of these enzymes turn off the proinflammatory chemokine signals.

\section{Proteinases as antimicrobial agents}

Proteinases have not traditionally been thought to play a significant role in host defence although some attention has gone to neutrophil serine proteinases. The recent finding by WILSON et al. [10] that gut epithelial cell MMP-7 induces activation of defensins was the first evidence that MMPs may be indirectly involved in antimicrobial activity. The role and abundance of epithelial cell-derived defensins in the lung has been questioned. The author's group propose that macrophages and their product MMP-12 play a significant role as the initial line of host defence when bacteria escape upper airway defenses and arrive in the alveolar space.

\section{Neutrophil serine proteinases}

When neutrophils encounter bacteria they internalise them within phagosomes that fuse to lysosomes exposing them to oxidative and nonoxidative mechanisms of killing. Proteinases and antimicrobial peptides termed defensins represent the major nonoxidative pathway. Serine proteinases, particularly NE, are becoming increasingly recognised for their importance in eliminating bacteria. The role of NE was first suspected three decades ago when JANOFF and BLONDIN [11] showed that NE extracts can lyse cell walls of heat-killed bacteria. Yet, the role of $\mathrm{NE}$ in bacterial killing was largely ignored until recently. GANZ and co-workers [12, 13] found that porcine polymorphonuclear leukocytes generate extracellular microbicidal activity by elastase-mediated activation of secreted proprotegrins. Mice deficient in NE were generated and it was demonstrated that NE is required for maximal killing of Gram-negative but not Gram-positive bacteria [14, 15]. NE kills Gram-negative bacteria via catalysis of specific outer membrane proteins in the outer membrane forming "holes" in the bacterial wall and allowing NE access to other intracellular bacterial target proteins [16]. NE also degrades bacterial toxins, protecting the neutrophil from their adverse effects [17].

\section{Matrix metalloproteinases as antimicrobial agents}

As mentioned above, there is limited data regarding the role of MMPs in antimicrobial killing. As a negative control for NE-mediated killing, MMP-9 was exposed to bacteria without effect on the growth of Gram-negative or Grampositive bacteria. As shown in preliminary data, the author's group has been unable to detect a direct ability of any major MMP to limit bacterial growth with the exception of MMP-12. MMP-7, while not directly antimicrobial, is able to activate defensins in the gut. As opposed to wildtype mice, extracts from the gastrointestinal (GI) tract of MMP-7-/- mice did not possess activated defensins. Moreover, MMP-7-/mice had altered flora and poorer clearance of bacteria instilled in the GI tract.

\section{Conclusion}

Preliminary data shows that MMP-12 represents the first MMP with direct antimicrobial activity. In the absence of MMP-12, septic mice have markedly increased bacterial loads in the lung. MMP-12-/- mice have a poorer survival in response to models of Gram-positive pneumonia. It has also been demonstrated that macrophages require MMP-12 for maximal intracellular bacterial killing.

This study represents the first intracellular function identified for a matrix metalloproteinase. Finally, these studies also strongly support the importance of the lung macrophage in the initial host defence against bacteria.

\section{References}

1. Hautamaki RD, Kobayashi DK, Senior RM, Shapiro SD. Requirement for macrophage elastase for cigarette smokeinduced emphysema in mice. Science 1997; 277: 2002-2005.

2. Zheng $\mathrm{T}$, Zhu Z, Wang Z, et al. Inducible targeting of IL-13 to the adult lung causes matrix metalloproteinase- and cathepsin-dependent emphysema. J Clin Invest 2000; 106: 1081-1093.

3. Wang Z, Zheng T, Zhu Z, et al. Interferon $\gamma$ induction of pulmonary emphysema in the adult murine lung. $J$ Exp Med 2000; 192: 1587-1599.

4. Lanone S, Zheng T, Zhu Z, et al. Overlapping and enzymespecific contributions of matrix metalloproteinase (MMP)-9 and MMP-12 to the pathogenesis of IL-13-induced inflammation and alveolar remodeling in the lung. $J$ Clin Invest 2002; 110: 463-474.

5. Wert S, Yoshida M, LeVine A, et al. Increased metalloproteinase activity, oxidant production, and emphysema in surfactant protein D gene-inactivated mice. Proc Natl Acad Sci USA 2000; 97: 5972-5977.

6. Morris DG, Huang X, Kaminski N, et al. Loss of integrinmediated TGF-beta activation causes MMP-12 dependent pulmonary emphysema. Nature 2003; 422: 169-173. 
7. Churg A, Wang RD, Tai H, et al. Macrophage metalloelastase mediates acute cigarette smoke-induced inflammation via tumour necrosis factor- $\alpha$ release. Am J Respir Crit Care Med 2003; 167: 1083-1089.

8. Li Q, Park PW, Wilson CL, Parks WC. Matrilysin shedding of syndecan-1 regulates chemokine mobilization and transepithelial efflux of neutrophils in acute lung injury. Cell 2002; 111: 635-646.

9. McQuibban GA, Gong JH, Tam EM, McCulloch CA, Clark-Lewis I, Overall CM. Inflammation dampened by gelatinase A cleavage of monocyte chemoattractant protein3. Science 2000; 289: 1202-1206.

10. Wilson C, Ouellette A, Satchell D, et al. Regulation of intestinal $\alpha$-defensin activation by the metalloproteinase matrilysin in innate host defense. Science 1999; 286: 113-117.

11. Janoff A, Blondin $\mathbf{J}$. The effect of human granulocyte elastase on bacterial suspensions. Lab Invest 1973; 29: 454457.

12. Panyutich A, Shi J, Boutz PL, Zhao C, Ganz T. Porcine polymorphonuclear leukocytes generate extracellular microbicidal activity by elastase-mediated activation of secreted proprotegrins. Infect Immun 1997; 65: 978-985.

13. Shi J, Ganz T. The role of protegrins and other elastaseactivated polypeptides in the bactericidal properties of porcine inflammatory fluids. Infect Immun 1998; 66: 3611-3617.

14. Belaaouaj A, McCarthy R, Baumann M, et al. Mice lacking neutrophil elastase reveal impaired host defense against gram negative pathogens. Nature Med 1998; 4: 615-619.

15. Tkalcevic J, Novelli M, Phylactides M, Iredale JP, Segal AW, Roes J. Impaired immunity and enhanced resistance to endotoxin in the absence of neutrophil elastase and cathepsin G. Immunity 2000; 12: 201-210.

16. Belaaouaj A, Kim KS, Shapiro SD. Degradation of outer membrane protein A in Escherichia coli killing by neutrophil elastase. Science 2000; 289: 1185-1187.

17. Weinrauch Y, Drujan D, Shapiro SD, Weiss J, Zychlinsky A. Neutrophil elastase targets virulence factors of enterobaceria. Nature 2002; 417: 91-94. 